\title{
Prostatic Size Alteration
}

National Cancer Institute

\section{Source}

National Cancer Institute. Prostatic Size Alteration. NCI Thesaurus. Code C40938.

Prostatic Size Alteration involves a change in the dimensions of the accessory sex gland (prostate gland) of the male reproductive system that surrounds the neck of the bladder and urethra and secretes into seminal fluid acid phosphatase, citric acid, and proteolytic enzymes that liquefy coagulated semen. 